\title{
Production of Medium Chain Glycerides and Monolaurin from Coconut Acid Oil by Lipase-Catalyzed Reactions
}

\author{
Sumit Nand, Sarbani Gangopadhyay and Santinath GHosh* \\ Department of Chemical Technology, Oil Technology Division, University of Calcutta \\ (92, APC Road, Kolkata - 700 009, West Bengal, INDIA)
}

Edited by Y. Shimada, Osaka Mun. Tec. Res. Inst., and accepted May 18, 2004 (received for review March 11, 2004)

\begin{abstract}
Medium chain glycerides (MCGs) containing $\mathrm{C}_{8: 0}$ and $\mathrm{C}_{10: 0}$ fatty acids have got commercial importance by considering their medicinal and nutritional applications. Monolaurin is also a value added commercial product. Coconut acid oil, which is mainly utilized in soap manufacturing process, can be effectively utilized to produce MCGs and monolaurin by a combination of lipase-catalyzed hydrolysis and esterification. Coconut acid oil containing $68 \%$ free fatty acid (as lauric acid), and 31.4\% neutral glycerides are hydrolyzed by Candida rugosa lipase. The hydrolyzed acid oil was then subjected to steam distillation under atmospheric pressure to get a fraction (yield, $5 \%)$ rich in medium chain fatty acids $\left(\mathrm{C}_{8: 0}, 46.2 \%, \mathrm{C}_{10: 0}, 19.2 \%\right)$, and the residual fraction was fractionally distilled under vacuum to get a fraction rich in lauric acid (68.4\%). These two fractions were esterified with glycerol with Rhizomucor miehei (Lipozyme RM IM) lipase to produce MCGs from the first fraction and monolaurin from the second fraction.
\end{abstract}

Key words: medium chain glyceride, monolaurin, Rhizomucor miehei lipase, Candida rugosa

\section{Introduction}

Short chain fatty acids (SCFAs) and medium chain fatty acids (MCFAs) have got some special importance, as SCFAs and MCFAs provide a quick source of energy for infants and stressed adults (1). Medium chain triglycerides (MCTs) containing $\mathrm{C}_{8: 0}$ and $\mathrm{C}_{10: 0}$ fatty acids have also got commercial importance because MCTs go directly into the portal vein and are rapidly converted to energy (2). MCFAs have several distinguished characteristics, such as high oxidative stability, low melting point and viscosity, and high solubility in water. MCTs are mainly utilized as a nutritional supplement for patients suffering from malabsorption caused by intestinal resection and as a component in infant formula (3-5). In addition, they also used as a solvent or a carrier of lipophilic nutrients or drugs, such as vitamin $\mathrm{K}$ (6) and phospholipid (7).

Medium chain glycerides (MCGs) which is mainly a mixture of mono-, di- and triglycerides have specific application in the field of foods, pharmaceuticals and cosmetics. Medium chain monoglycerides are ideal solvent for aromatics, steroids, dyes and perfume bases. A mixture of medium chain monoglyceride and diglyceride was found to be an effective solvent for dissolving cholesterol gallstones in humans (8).

Commercially MCGs are manufactured by glycerolysis of MCFAs at high temperature and high pressure, followed by alkali refining, washing, molecular distillation and activated carbon treatment for the products.

Recently structured lipids that contain mixture of short or medium chain fatty acids or both and long

\footnotetext{
*Correspondence to: Santinath GHosh, Department of Chemical Technology, Oil Technology Division, University of Calcutta, 92 , APC Road, Kolkata - 700 009, West Bengal, INDIA

E-mail: santinathghosh@yahoo.com.hk
} 
chain fatty acids are beneficial in human nutrition because they can be tailor-made to metabolic conditions. Lipase-catalyzed modification of triglycerides (TGs) to produce various structured TGs containing MCFAs and long chain essential fatty acids have got tremendous commercial importance due to several advantages over chemical catalyzed process. Structured TGs have been produced by exchanging fatty acids in natural fats and oils with MCFAs using an immobilized 1,3-specific lipase by several workers (9-13). MCFA rich glycerides have been prepared from coconut oil and MCFA methyl esters by lipase catalyzed polyestermonoester interchange reaction (14).

Coconut acid oil, a by-product of coconut oil refinery industry, is mainly utilized in soap manufacturing process. Different value added products are produced from commercial acid oils by lipase catalyzed reactions (15). But no one has utilized the coconut acid oil as a source of cheap raw material to produce MCFAs. The aim of the present work is to utilize coconut acid oil to produce MCFAs and lauric acid, and subsequent production of MCGs and monolaurin by an enzymatic esterification with glycerol.

\section{Experimental}

\subsection{Material}

Coconut acid oil was obtained from Edible Products (I) Pvt. Ltd., Kolkata, India. The immobilized lipase Rhizomucor miehei (Lipozyme RM IM) was a gift of Novozyme South Asia Pvt. Ltd. Bangalore, India, Candida rugosa lipase was purchased from Sigma Chemical Co. USA, “Amano" 30 (Candida rugosa) lipase was a kind gift of AMANO ENZYME Inc., Nagoya, Japan.

\subsection{Enzymatic Hydrolysis of Coconut Acid Oil}

Acid oil $(100 \mathrm{~g})$ was taken in a $250 \mathrm{ml}$ stoppered Erlenmeyer flask and water ( $60 \%$ by weight of neutral glycerides) containing lipase powder $(0.4 \%$ Candida rugosa lipase or 1.0\% "Amano" 30 lipase) was added. The reaction mixture was magnetically stirred with a 1 inch teflon coated stir bar at $35 \pm 2^{\circ} \mathrm{C}$ in a controlled bath. The degree of hydrolysis was determined by the content of free fatty acid in the sample periodically withdrawn. After complete reaction, the oil layer and water layer containing enzyme and glycerol were sepa- rated by centrifugation.

\subsection{Atmospheric Steam Distillation}

The hydrolyzed coconut acid oil was subjected to steam distillation for $2 \mathrm{~h}$ under atmospheric pressure in an all glass distillation equipment fitted with steam inlet, steam outlet and thermometer pocket adopter. The steam volatile fatty acids were collected in the distillate.

\subsection{Fractional Distillation}

The residual fatty acids from the steam distillation were fractionally distilled in a Claisen-Vigroux (250 $\mathrm{ml}$ ) flask. The flask was connected to a vacuum pump through a distillation head, an air condenser and preweighed $100 \mathrm{ml}$ round bottom collecting flask. The volatile fraction from $100^{\circ} \mathrm{C}$ to $140^{\circ} \mathrm{C}$ at $4 \mathrm{mmHg}$ pressure was collected for $30 \mathrm{~min}$. The feed, distillate and residue fractions were weighed and analyzed.

\subsection{Enzymatic Esterification Reaction}

Fatty acids and glycerol in an appropriate proportion were taken in a round bottom flask and stirred by a magnetic stirrer at $60 \pm 2{ }^{\circ} \mathrm{C}$ for $8 \mathrm{~h}$ using $10 \%$ (by weight of fatty acids) RM IM lipase. The stopper of the flask was kept open to eliminate the water formed during esterification reaction. The esterification reaction was monitored by estimating the free fatty acid content in the samples periodically withdrawn. After complete reaction, the product mixture was isolated and mono-, di- and triglycerides, and their amounts were determined by column chromatographic method.

Fatty acid composition was determined by a gas-liquid chromatographic (GLC) method after converting the fatty acids into methyl esters. The HP-5890A GLC was connected with a HP-3390A data integrator. The GLC was fitted with a glass column $\left(6^{\prime} \times 1 / 8^{\prime \prime}\right.$ i.d. $)$, packed with $10 \%$ DEGS supported on ChromosorbWHP (100/200 mesh), of HP make. The oven temperature was programmed from $100^{\circ} \mathrm{C}$ to $190^{\circ} \mathrm{C}$ at $5^{\circ} / \mathrm{min}$. The injector, and detector block temperatures were maintained at $230^{\circ} \mathrm{C}$, and $240^{\circ} \mathrm{C}$, respectively. IOLAR-2 nitrogen was used as the carrier gas (flow rate, 30 $\mathrm{mL} / \mathrm{min}$ ). The fatty acid ester peaks were identified and calibrated with standard methyl esters. Data are averages of three determinations. 


\section{Results and Discussion}

Table 1 shows the total fatty acid composition, unsaponifiable matter, and neutral glycerides content of the coconut acid oil. Neutral glycerides present in the acid oil is a mixture of mono-, di- and triglycerides. The acid oil contains $4.9 \%$ caprylic acid $\left(\mathrm{C}_{8: 0}\right)$ and $6.2 \%$ capric acid $\left(\mathrm{C}_{10: 0}\right)$. So about $11 \%$ MCFAs are present.

The neutral glycerides present in acid oil were hydrolyzed by Candida rugosa lipase as shown in Fig. 1. After $12 \mathrm{~h}$, the degree of hydrolysis reached about $96 \%$. The hydrolyzed acid oil was then subjected to steam distillation to get a fraction (fraction I) rich in MCFAs $\left(\mathrm{C}_{8: 0}\right.$ and $\left.\mathrm{C}_{10: 0}\right)$. The residual fraction was then fractionally distilled under vacuum to get a fraction (fraction II) rich in lauric acid. Table 2 shows the yield and fatty acid composition of the two fractions and the residue. The yield of fraction I was $5 \%$, which contained $8.2 \%$ caproic acid $\left(\mathrm{C}_{6: 0}\right), 46.2 \%$ caprylic acid $\left(\mathrm{C}_{8: 0}\right)$ and $19.2 \%$ capric acid $\left(\mathrm{C}_{10: 0}\right)$. So fraction I can be used as a source of MCFAs. Fraction II (yield 63\%) contained mainly $68.4 \%$ lauric acid $\left(\mathrm{C}_{12: 0}\right)$ and $17.1 \%$ myristic acid $\left(\mathrm{C}_{14: 0}\right)$. So fraction II can be used as a source of lauric acid.

Table 1 Analytical Characteristics and Fatty Acid Composition of Coconut Acid Oil.

\begin{tabular}{lc}
\hline \multicolumn{1}{c}{ Properties } & $(\% \mathrm{w} / \mathrm{w})$ \\
\hline FFA(as lauric acid) & 68.5 \\
Unsaponifiable matter & 0.3
\end{tabular}

Neutral glycerides

$\begin{array}{lr}\text { Monoglycerides } & 4.3 \\ \text { Diglycerides } & 3.6 \\ \text { Triglycerides } & 23.3\end{array}$

Fatty acid Composition (total)

\begin{tabular}{lr}
$\mathrm{C}_{6: 0}$ & 0.9 \\
$\mathrm{C}_{8: 0}$ & 4.9 \\
$\mathrm{C}_{10: 0}$ & 6.2 \\
$\mathrm{C}_{12: 0}$ & 50.3 \\
$\mathrm{C}_{14: 0}$ & 19.2 \\
$\mathrm{C}_{16: 0}$ & 8.3 \\
$\mathrm{C}_{18: 0}$ & 1.3 \\
$\mathrm{C}_{18: 1}$ & 6.3 \\
$\mathrm{C}_{18: 2}$ & 2.6 \\
\hline
\end{tabular}

Fraction I and II were then esterified with glycerol using 1,3-specific RM IM lipase to get MCGs and monolaurin, respectively. The fraction I fatty acids were esterified with glycerol to produce a mixture of MCGs and the reaction was monitored by determining free fatty acid (FFA) with time (Fig. 2). After 8 h, the FFA in the mixture was only $6.5 \%$. So most of the fatty acids were esterified with glycerol after $8 \mathrm{~h}$. The final product contained $18.9 \%$ monoglycerides (MGs), $62.4 \%$ diglycerides (DGs) and $12.2 \%$ TGs (Table 3). The higher amount of DGs in the reaction mixture was due to the specificity of the lipase (1,3-position specific). TGs were produced due to some acyl migration from the 1,3-positions to 2 position.

Fraction II was also esterified with glycerol by taking larger amount of glycerol to efficiently produce MGs

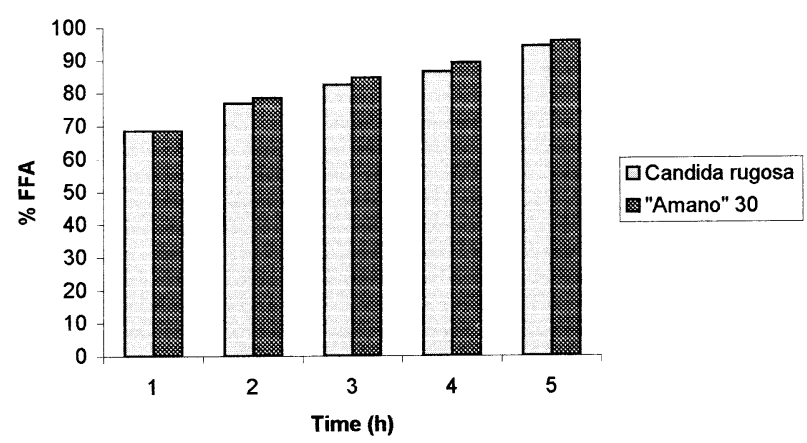

Fig. 1 Hydrolysis of Coconut Acid Oil.

Table 2 Yield and Fatty Acid Composition of the Fractions.

\begin{tabular}{lcccc}
\hline & & $\begin{array}{l}\text { Fraction I } \\
\text { (by steam } \\
\text { distillation) }\end{array}$ & $\begin{array}{l}\text { Fraction II } \\
\text { (by fractional }\end{array}$ & Residue \\
distillation) & \\
\hline Yield $(\%, \mathrm{w} / \mathrm{w})$ & & 5 & 63 & 32 \\
& & & & \\
Fatty acid & $\mathrm{C}_{6: 0}$ & 8.2 & 0.7 & 0 \\
composition & $\mathrm{C}_{8: 0}$ & 46.2 & 3.2 & 1.8 \\
& $\mathrm{C}_{10: 0}$ & 19.2 & 5.0 & 6.5 \\
& $\mathrm{C}_{12: 0}$ & 23.0 & 68.4 & 20.5 \\
& $\mathrm{C}_{14: 0}$ & 3.4 & 17.1 & 24.4 \\
& $\mathrm{C}_{16: 0}$ & - & 4.5 & 17.0 \\
& $\mathrm{C}_{18: 0}$ & - & 0.4 & 3.1 \\
& $\mathrm{C}_{18: 1}$ & - & 0.7 & 18.6 \\
& $\mathrm{C}_{18: 2}$ & - & 0 & 8.1 \\
\hline
\end{tabular}




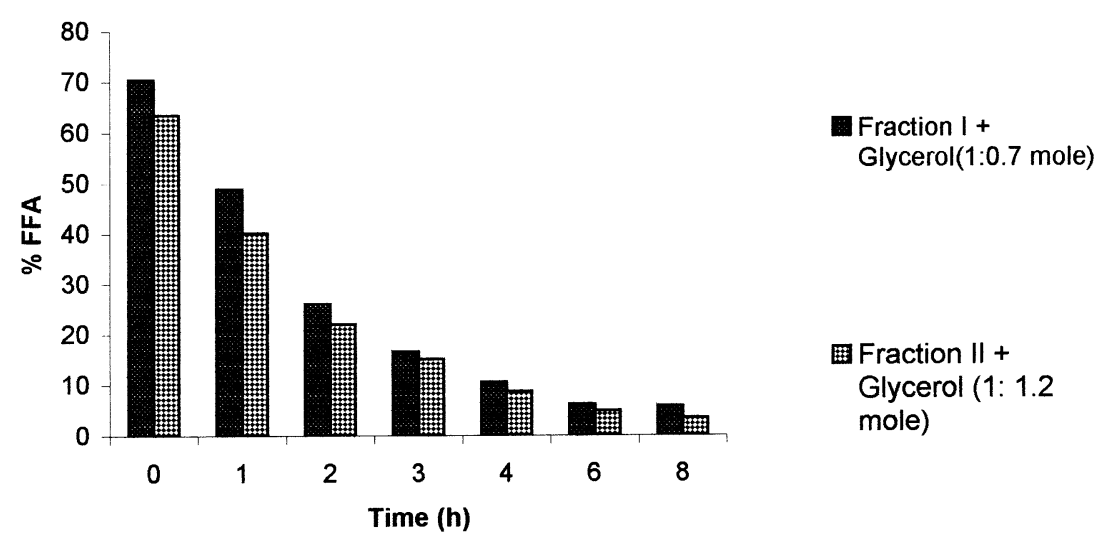

Fig. 2 Esterification of the Distilled Fatty Acids with Glycerol.

Table 3 Composition of the Medium Chain Glycerides and Monolaurin.

\begin{tabular}{lcccr}
\multicolumn{5}{c}{ Composition $(\% \mathrm{w} / \mathrm{w})$} \\
\hline & FFA & MG & DG & TG \\
\hline Medium Chain Glycerides from Fraction I & 6.5 & 18.9 & 62.4 & 12.2 \\
Monolaurin from Fraction II & 4.2 & 55.3 & 36.9 & 3.6 \\
\hline
\end{tabular}

(Table 2 and 3). The product (monolaurin) contained 4.2\% FFAs, 55.3\% MGs, 36.9\% DGs and 3.6\% TGs.

\section{Conclusion}

It can be noted that the microbial lipase technology with a non-specific lipase offers excellent means of producing fatty acids from coconut acid oil. The valuable MCFAs were produced from coconut acid oil. MCFAs and lauric acid were readily converted into MCGs and monolaurin by lipase-catalyzed esterification. Microbial lipase may be preferred to the chemical catalyst process in producing better quality of the concerned products from the relatively inferior grade raw material.

\section{Acknowledgements}

The research was financially supported by a research grant from Department of Biotechnology, Govt. of India.

\section{References}

1. W.G. HEIRD, S.M. GRUNDY and V.S. HUBBARD, Structured Lipids and Their Use in Clinical Nutrition. Workshop at the
National Institutes of Health, Bethesda, Maryland, Am. J. Clin. Nutr., Vol. 43, 320-324 (1986).

2. A.C. BACH and V.K. BABAYAN, Medium-Chain Triglycerides: An Update, Am. J. Clin. Nutr., Vol. 36, 950-961 (1982).

3. V.K. BABAYAN, Medium-Chain Length Fatty Acid Esters and Their Medical and Nutritional Applications, J. Am. Oil Chem. Soc., Vol. 58, 49A-51A (1981).

4. M. YAMASHITA and Y. KADONA, Digestion, Absorption, and Metabolism of Medium-Chain Triglycerides, New Food Industry, Vol. 24, 28-33 (1982).

5. U. JULIUS and W. LEONHARDT, Elimination and Metabolism of a Fat Emulsion Containing Medium-Chain Triglycerides (lipofundin MCT 10\%), J. Parenteral and Enternal Nutrition, Vol. 12, 116-120 (1988).

6. Snow Brand Milk Products, Japanese Patent 85922 (1988).

7. Shojiikk Sanyu, Japanese Patent, 049747 (1985).

8. S.M. KIM and J.S. RHEE, Production of Medium-Chain Glycerides by Immobilized Lipase in a Solvent-Free System, J. Am. Oil Chem. Soc., Vol. 68, 499-501 (1991).

9. I.H. KIM, H. KIM, K.T. LEE, S.H. CHUNG and S.N. KO, Lipase-Catalysed Acidolysis of Perilla Oil with Caprylic Acid to produce Structured Lipids, J. Am. Oil Chem. Soc., Vol. 79, 363366 (2002).

10. A. KAWASHIMA, Y. SHIMADA, T. NAGAO, A. OHARA, T. MATSUHISA, A. SUGIHARA and Y. TOMINAGA, Production of Structured TAG Rich in 1,3-Dicapryloyl-2- $\gamma$-linolenoyl Glycerol from Borage Oil, J. Am. Oil Chem. Soc., Vol. 79, 871-877 (2002). 
11. R. IRIMESCU, Y. IWASAKI and C.T. HOU, Study of TAG to 2MAG by Immobilized Candida antartica Lipase and Synthesis of Symmetrically Structured TAG, J. Am. Oil Chem. Soc., Vol. 68, 879-883 (2002).

12. U. SCHMID, U.T. BORNSCHEUER, M.M. SOUMANOU, G.P. MCNEILL and R.D. SCHMID, Optimization of the Reaction Conditions in the Lipase-Catalyzed Synthesis of Structured Triglycerides, J. Am. Oil Chem. Soc., Vol. 75, 1527-1531 (1998).

13. X. XU, T. PORSGAARD, H. ZHANG, J. ALDER-NISSEN and C. HOY, Production of Structured Lipids in a Packed-Bed Reac- tor with Thermomyces lanuginosa Lipase, J. Am. Oil Chem. Soc., Vol. 79, 561-565 (2002).

14. S. GHOSH and D.K. BHATTACHARYYA, Medium-Chain Fatty Acid-Rich Glycerides by Chemical and Lipase-Catalyzed Polyester-Monoester Interchange Reaction, J. Am. Oil Chem. Soc., Vol. 74, 593-595 (1997).

15. S. GHOSH and D.K. BHATTACHARYYA, Utilization of Acid Oils in Making Valuable Fatty Products by Microbial Lipase Technology, J. Am. Oil Chem. Soc., Vol. 72, 1541-1544 (1995). 